\title{
Correspondance
}

\section{Treatment for otitis media}

T $t$ is a pity that the otherwise superb 1 paper by Nicole Le Saux and associates $^{1}$ is marred by some mangled syntax. If after reading a sentence 3 times you are still uncertain that you have understood it correctly, then the sentence is badly written. I guessed at the meaning of the first sentence in the Interpretation section of the abstract for this paper, but it might help if the authors could remove at least one negative so that I can verify whether I guessed correctly. And what about the term "noninferiority" in the article title and elsewhere? Why not "equivalence" or, better still, simply refer to "a randomized, double-blind, placebo-controlled trial"? Readers are likely to be less intimidated once the unfamiliar word is removed.

This nitpicking aside, the study is exemplary. However, it will be interesting to see whether the favourable results for fever and pain on days 1 and 2 will persuade parents to prefer an antibiotic over watchful waiting.

\section{Barry Pless}

Director, Clinical Research

Montreal Children's Hospital Research Institute

Editor, Injury Prevention

Montréal, Que.

\section{Reference}

1. Le Saux N, Gaboury I, Baird M, Klassen TP MacCormick J, Blanchard C, et al. A randomized, double-blind, placebo-controlled noninferiority trial of amoxicillin for clinically diagnosed acute otitis media in children 6 months to 5 years of age. CMAf 2005;172(3):335-41.

Competing interests: None declared.

DOI:10.1503/cmaj.1050041

$\mathrm{F}$ or many people, the concept of noninferiority studies may not be intuitive. It is therefore of concern that the article cited by Nicole Le Saux and associates $^{1}$ as methodologic support for their study design did not explicitly mention the term "noninferiority" or "margin of noninferiority," although it did include the operational definition. Equivalence and noninferiority studies are usually considered related, yet distinct epidemiologic entities. Indeed, "equivalence trials ... aim to match the action of an established therapy and prove this to statistical significance within a predetermined range ([delta]), in both positive and negative directions (a 2-sided test) ... [whereas] noninferiority trials are statistically based on a 1sided comparison to an active control in the positive direction (a 1-sided test)."

It is unclear to me whether, on theoretical grounds, this was an appropriate methodologic choice for the amoxicillin study. "[E]quivalence and noninferiority trials rely on the premises that the superior efficacy of the active control over placebo has been previously proven for a given indication, and that this efficacy will be preserved under the conditions of the trial. If either of these assumptions is not valid, the results become suspect unless the primary analysis demonstrates differences between the experimental and active-control treatments." Since the conclusion of Le Saux and associates may also be a necessary assumption for the internal validity of the trial, perhaps this trial was a "self-fulfilling prophecy."

Furthermore, "a fundamental assumption in the design and analysis of an active-control noninferiority trial is that the active control is truly effective." ${ }^{4}$ This assertion suggests the following corollary for this study: amoxicillin must be superior to placebo (i.e., has proven efficacy). Since the investigators set out to determine whether placebo was noninferior to amoxicillin, one has to assume that it was still unclear whether the drug was superior. Therefore, the apparently crucial premise of proven efficacy of the active control (amoxicillin) was not met.

Unless the investigators can provide a satisfactory explanation for these apparent illogicalities, one additional question remains: To what extent does such a discrepancy affect their conclusions?

\section{Mathieu Lemaire}

Pediatric Resident

The Hospital for Sick Children

Toronto, Ont.

\section{References}

1. Le Saux N, Gaboury I, Baird M, Klassen TP, MacCormick J, Blanchard C, et al. A randomized, double-blind, placebo-controlled noninferiority trial of amoxicillin for clinically diagnosed acute otitis media in children 6 months to 5 years of age. CMA7 2005;172(3):335-41.

2. Jones B, Jarvis P, Lewis JA, Ebbutt AF. Trials to assess equivalence: the importance of rigorous methods [published erratum appears in $B M \mathcal{F}$ 1996;313(7056):550]. BM7 1996;313(7048):36-9.

3. Gomberg-Maitland M, Frison L, Halperin JL. Active-control clinical trials to establish equivalence or noninferiority: methodological and statistical concepts linked to quality. Am Heart 7 2003;146(3):398-403.

4. Chen G, Wang YC, Chi GY. Hypotheses and type I error in active-control noninferiority trials. F Biopharm Stat 2004;14(2):301-13.

Competing interests: None declared.

DOI:10.1503/cmaj.1050050

$\mathrm{N}$ icole Le Saux and associates ${ }^{1}$ report that amoxicillin is superior to placebo in relieving effusion and symptoms of acute otitis media and review the current controversy surrounding the "do not treat" philosophy.

I believe that the problem lies with the diagnosis. We physicians have become too inclusive in our diagnosis of "acute" - that is, bacterial — otitis media. Including patients with noninfected effusion under the umbrella of acute otitis media has led to overtreatment. The number of patients with actual signs or symptoms of acute inflammation in the ear is actually quite low. I recently had the opportunity to confirm this in a survey of Manitoba pediatricians, family physicians, emergency physicians and otolaryngologists (unpublished data). No wonder some authors find that treatment is not effective.

\section{Brian W. Blakley}

Professor and Chairman

Department of Otolaryngology

University of Manitoba

Winnipeg, Man.

\section{Reference}

1. Le Saux N, Gaboury I, Baird M, Klassen TP, MacCormick J, Blanchard C, et al. A randomized, double-blind, placebo-controlled noninferiority trial of amoxicillin for clinically diagnosed acute otitis media in children 6 months to 5 years of age. CMA7 2005;172(3):335-41.

Competing interests: None declared.

DOI:10.1503/cmaj.1050054 